\title{
Análisis de las caídas en domicilio de los pacientes en hemodiálisis
}

\author{
Luis Ignacio Latorre López, Aida Delgado Ramírez, Estíbaliz Ruiz García \\ Facultad de Medicina y Enfermería de Córdoba. Unidad de Gestión Clínica de Nefrología. Hospital Universitario Reina \\ Sofía de Córdoba. España
}

\section{Resumen}

Introducción: Los enfermos con enfermedad renal crónica avanzada en hemodiálisis son cada vez mayores. Además de la edad, presentan una alta comorbilidad asociada que, unido a una serie de alteraciones en el metabolismo óseo-mineral propios de la enfermedad, condiciona que sean una población especialmente frágil ante las caídas.

Objetivos: Analizar la incidencia y características de las caídas de los pacientes con enfermedad renal crónica avanzada en hemodiálisis fuera de la unidad de diálisis.

Pacientes y Métodos: Se estudiaron 60 pacientes con una edad media de $66.6 \pm 14.2$ años (70\% hombres, $30 \%$ mujeres).Del total de la muestra, 27 pacientes (45\%) tuvieron alguna caída en el último año.

Se encontraron diferencias significativas entre los pacientes que tuvieron caídas frente a los que no ( $p=0.017$ ) en el tiempo en diálisis. Un $37 \%$ de los hombres habían sufrido caídas mientras que las mujeres que se habían caído fueron el $61 \%$.

Un 59\% de las caídas tuvieron lugar en el propio domicilio, el $22 \%$ en la calle, el $8 \%$ en el hospital. Respecto al día de la caída el $41 \%$ de las caídas se produjeron el día de descanso, el $22 \%$ antes o después de la sesión y el $15 \%$ no lo recuerdan.

Correspondencia:

Luis Ignacio Latorre López

Carretera de las Ermitas n 8314012 Córdoba

latorrerus@gmail.com
Conclusiones: Nuestros resultados indican que las caídas de los pacientes en hemodiálisis tienen una incidencia alta. Estas caídas están relacionadas con ser mujer, llevar más tiempo en diálisis, tener mayor grado de dependencia o realizar menor actividad física; y se producen mayoritariamente en el domicilio del paciente el día de descanso de diálisis.

\section{PALABRAS CLAVE}

- CAÍDAS

- HEMODIÁLISIS

- FRÁGIL

- INSUFICIENCIA RENAL CRÓNICA

\begin{abstract}
Introduction: Patients with advanced chronic kidney disease on hemodialysis are increasing. Apart from age, they have a high comorbidity that together with a series of alterations in bone-mineral metabolism associated with the disease cause a greater propensity to fall.

Objectives: To analyze the incidence and characteristics of falls in patients with advanced chronic kidney disease on hemodialysis outside the dialysis unit.

Patients and Methods: We studied 60 patients with a mean age of $66.6 \pm 14.2$ years $(70 \%$ men, $30 \%$ women). From the total sample, 27 patients (45\%) had a fall in the last year.

Significant differences between patients who had fallen versus those who had not fallen $(p=0.017)$ on the dialysis time were found. $37 \%$ of men had suffered falls while women who had fallen were $61 \%$.
\end{abstract}


$59 \%$ of falls occurred in the home, $22 \%$ in the street and $8 \%$ in the hospital. Regarding the day of the fall, $41 \%$ of falls occurred the day of rest, $22 \%$ before or after the session and $15 \%$ did not remember.

Conclusions: Our results indicate that falls in hemodialysis patients have a high incidence. These falls are related to being a woman, staying longer on dialysis, have a greater degree of dependence or perform less physical activity. Falls occur mainly in the patient's home and the rest day.

\section{KEYWORDS}

- FALLS

- HEMODIALYSIS

- FRAIL

\section{Introducción}

El aumento de la esperanza de vida en los países industrializados está produciendo un aumento de la población anciana en los mismos y esta población se caracteriza por sufrir un aumento de las patologías crónicas lo que conlleva un declive físico y psíquico que condiciona su capacidad funcional, siendo cada vez mayor el número de personas que precisan de cuidados $^{1-3}$. El envejecimiento fisiológico se ha relacionado con procesos de inflamación, pérdida de la densidad ósea y la presencia de enfermedad vascular aterosclerótica. Se ha reconocido que, como parte de este proceso, existe un leve declive de la función física y cognitiva, así como de las funciones metabólicas ${ }^{4}$.

Una población con patología crónica y con comorbilidad asociada son los pacientes con enfermedad renal crónica avanzada (ERCA), cuya prevalencia está aumentando progresivamente debido al envejecimiento de la población ${ }^{5}$. En efecto, según el Registro de la Sociedad Española de Nefrología de 2014, la mayoría de población con ERCA con terapia renal sustitutiva (TRS) tiene una edad superior a 65 años 6 . Además, el TRS es un componente muy importante del gasto de salud, dado que, aunque el volumen de enfermos es menor del $0,1 \%$ de la población, el presupuesto de salud es del 2,5\% para esta población ${ }^{7}$.

Por tanto, los enfermos con ERCA están aumentando progresivamente con tasas anuales del $5-8 \%$ de crecimiento en los países desarrollados, siendo estos datos considerados como epidémicos ${ }^{8}$. Además, los pacientes en TRS con hemodiálisis (HD) aparte de la edad, van a presentar una serie de alteraciones inherentes a la enfermedad renal9,10.

Por otra parte, la edad se ha asociado con un mayor riesgo de sufrir caídas. Se han definido las caídas como acontecimientos involuntarios que hacen perder el equilibrio y dar con el cuerpo en tierra u otra superficie firme que lo detenga ${ }^{11}$. Se calcula que un tercio de los mayores 64 se caerán en un año y un $15 \%$ se volverán a caer ${ }^{12}$. Las caídas suelen afectar a la salud negativamente provocando un cese de las actividades de la vida diaria, algún tipo de fractura o incluso la muerte.

Teniendo en cuenta, que la ERCA presenta una serie de trastornos asociados tales como anemia, alteraciones osteominerales, inflamación y malnutrición, cabe pensar que esta población además de fragil ${ }^{13}$ puede ser especialmente sensible a las caídas.

En este sentido, se ha señalado que los pacientes con ERCA en tratamiento con HD, tienen más riesgo de caerse que la población en general debido a la polimedicación, a la comorbilidad y los cambios en su volumen plasmático ${ }^{14}$.

Por todo ello, nos propusimos el análisis cronológico de las caídas en pacientes en HD para comprobar cuáles son los factores asociados a estas y cuáles son sus características.

\section{Objetivos}

Analizar la incidencia y características de las caídas fuera de la unidad de diálisis de los pacientes con ERCA en tratamiento con HD.

\section{Pacientes y métodos}

\section{Ámbito del estudio}

El estudio se desarrolló en la Unidad de Gestión Clínica (UGC) Nefrología del Hospital Universitario Reina Sofía (HURS) de Córdoba, previa autorización del director de la UGC y con la colaboración del personal de enfermería de esta UGC.

\section{Población y muestra}

La población de estudio han sido los pacientes con ERCA en HD periódicas de la Unidad Hospitalaria y del Centro Periférico de Diálisis de la UGC Nefrología 
del HURS. Se realizó un muestreo no probabilístico accidental incluyendo a todos los pacientes que aceptaron colaborar en este estudio después de leer la hoja informativa y firmar el consentimiento informado. Se incluyeron en la muestra un total de 60 pacientes, 42 (70\%) hombres y $18(30 \%)$ mujeres.

\section{Criterios de inclusión}

Pacientes de ambos sexos en HD, que aceptaron participar en el estudio tras firmar el consentimiento informado.

\section{Diseño}

Se realizó un estudio observacional, descriptivo y transversal.

\section{Variables del estudio}

Las variables demográficas añadidas en el estudio fueron: edad, género, tiempo en diálisis, actividad física, actividades para la vida diaria (AVD), y enfermedad de base y toma medicación antihipertensiva, de los pacientes que habían tenido alguna caída. Estas variables se obtuvieron en la entrevista personal, sin tener que acceder a la historia clínica del paciente.

La variable de resultado fue haber tenido alguna caída fuera de la unidad de diálisis en el último año: causa, fecha (estación), hora, características, lugar y día en relación con la sesión de HD. No se consideró el número de caídas por paciente.

\section{Instrumento de medida}

Se utilizó un cuestionario de elaboración propia que indagaba en el número de caídas, así como todo lo relacionado con las mismas (Anexo 1 ). Previamente a iniciar el estudio se realizó una prueba piloto para comprobar la idoneidad de las preguntas para el objetivo planteado, entrevistando a algunos pacientes, para comprobar la validez de las respuestas.

Para el análisis de las AVD se utilizó el Índice de Barthe ${ }^{15}$.

\section{Recogida de los datos}

Los datos se recogieron en el periodo comprendido entre el 12-30 de Abril de 2016, fecha que sirvió además de referencia para el periodo en el que se habían producido las caídas (un año hacia atrás); y se realizaron durante las sesiones de HD de los pacientes en las dos unidades de diálisis, sin que fuera necesario añadir tiempo extra para la cumplimentación de los mismos.

\section{Aspectos éticos}

La información obtenida ha sido utilizada exclusivamente para los fines de esta investigación y es totalmente confidencial y tratada conforme a la Ley Orgánica 15/1999 de 13 diciembre, sobre "Protección de datos de carácter personal". Si en algún momento el paciente deseó retirarse del estudio, lo pudo hacer sin tener que dar ningún tipo de explicación. Se garantizó en todo momento el anonimato de las pacientes.

El estudio se inició después de recibir el informe favorable del Comité de Ética e Investigación del HURS.

\section{Análisis estadístico}

Para las variables cualitativas se utilizó una distribución de frecuencias, mientras que para las variables numéricas se usó la media \pm la desviación estándar de la media y en algunos casos la mediana. Para la comparación entre variables se utilizó la t de Student para medias independientes y la $\mathrm{U}$ de Mann-Whitney según siguieran o no una distribución normal. Se aceptó significación estadística para $p<0.05$. El análisis estadístico se ha realizado en un paquete estadístico SSPS 15.0 para Windows.

\section{Resultados}

Se estudiaron 60 pacientes con una edad media de $66.6 \pm 14.2(32-93)$ años. El $70 \%$ eran hombres y el $30 \%$ mujeres. 24 meses y una amplitud intercuartil (AI) de 59 meses. En cuanto a la actividad física, el 30\% manifestó realizar una actividad mínima, el $65 \%$ una actividad moderada y el $5 \%$ una actividad vigorosa. En cuanto a la capacidad de los pacientes para llevar a cabo las AVD con el Índice de Barthel, se representan Tabla 1. Hay que indicar que no se encontró ningún paciente Dependiente Absoluto, según las categorías de este índice.

Tabla 1. Distribución de los pacientes según el grado de dependencia.

\begin{tabular}{lcc} 
Categorías & Frecuencia & Porcentaje \\
\hline Independientes & 29 & $48 \%$ \\
Dependencia escasa & 7 & $12 \%$ \\
Dependencia moderada & 20 & $33 \%$ \\
Dependencia severa & 4 & $7 \%$ \\
\hline
\end{tabular}


Del total de la muestra estudiada, 27 pacientes tuvieron alguna caída, lo que representa al $45 \%$ del total ( 16 hombres y 11 mujeres). Respecto a la actividad física que decían realizar a diario, encontramos que los que realizaban una mínima actividad se han caído $61 \%$, los que realizaban actividad moderada el $41 \%$, y los que realizaban una actividad vigorosa no se habían caído ninguno en el tiempo estudiado.

En cuanto a las AVD y su relación con las caídas, podemos observar en la Tabla 2 la distribución de frecuencias entre los que han sufrido o no caídas en las diferentes categorías del Índice de Barthel.

Tabla 2. Distribución del grado de dependencia en función de las caídas.

\begin{tabular}{|lcc|}
\hline Categorías Barthel & SI & NO \\
\hline Independientes & $27 \%$ & $73 \%$ \\
\hline Dependencia escasa & $71 \%$ & $29 \%$ \\
Dependencia moderada & $50 \%$ & $50 \%$ \\
\hline Dependencia severa & $100 \%$ & $0 \%$ \\
\hline
\end{tabular}

Cuando se analizó la edad en función de las caídas, la edad media de los pacientes que tuvieron caídas fue de $69 \pm 11.5$ años, frente a los que no habían tenido caídas que fue de $64.6 \pm 15.8$ años, no siendo estas diferencias estadísticamente significativas. De igual forma, cuando se compara el tiempo en HD, los pacientes que tuvieron caídas, tenían una mediana de 48 meses $(72$, AI) y los que no habían tenidos caídas de 18 meses (54, AI), siendo esta diferencias estadísticamente significativas $(p=0.017)$.

Al analizar el género de los pacientes que habían tenido una caída en relación a la muestra total estudiada, obtuvimos que los hombres que habían sufrido caídas fueron el $37 \%$ respecto al total de hombres, mientras que las mujeres que habían sufrido caídas representaban el $61 \%$ del total de mujeres.

Cuando se analizaron las características de las caídas se establecieron 4 categorías para clasificar la causa de las mismas, como puede apreciarse en la Tabla 3.

Tabla 3. Clasificación de las causas de las caídas.

\begin{tabular}{lc}
\hline Causas & Porcentaje \\
\hline Accidental & $59 \%$ \\
Hipotensión/ mareo / hipoglucemia & $22 \%$ \\
Pérdida de fuerza / debilidad piernas & $11 \%$ \\
Problema visión / otros problemas médicos & $8 \%$ \\
\hline
\end{tabular}

Respecto a la enfermedad de base de los pacientes que habían tenido alguna caída se representa la siguiente tabla (Tabla 4).

Tabla 4. Distribución de la enfermedad de base en los pacientes que habían sufrido caídas.

\begin{tabular}{|lc|}
\hline Categorías & Porcentaje \\
\hline Diabetes Tipo I y II & $26 \%$ \\
\hline Glomerulonefritis, Pielonefritis & $15 \%$ \\
\hline Enfermedad vascular renal & $7 \%$ \\
Enfermedad poliquística, tumor renal & $15 \%$ \\
\hline Etiología desconocida & $37 \%$ \\
\hline
\end{tabular}

En cuanto a la estación del año en la que se habían caído, 6 pacientes creían que había sido en invierno, 6 en primavera, 4 en verano, uno en otoño y 10 no lo recordaban. Sobre el rango horario en el que supuestamente se habían caído, 6 pacientes manifestaron haberse caído por la mañana, 4 al mediodía, 3 por la tarde y uno en la noche.

Las caídas se produjeron en el propio domicilio del paciente en el $59 \%$ de los casos, en el hospital (fuera de la unidad de diálisis) en el $8 \%$, en la calle en el $22 \%$ y el $11 \%$ de los pacientes no lo recordaba.

En el Gráfico 1 está representada la distribución del día de la caída en relación al día en el que se dializaban los pacientes.

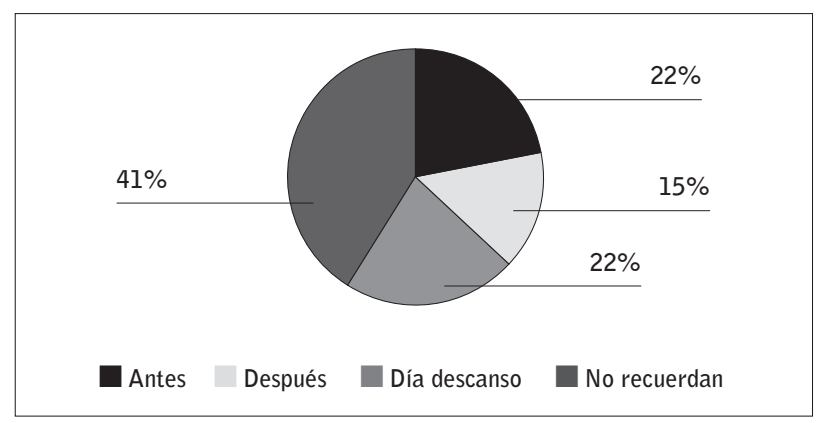

Gráfico 1. Día en el que caen los pacientes en relación a la sesión de HD.

Respecto a la toma de medicación antihipertensiva de los pacientes que había sufrido alguna caída, el $56 \%$ si tomaba esta medicación, el $37 \%$ no la tomaba, y el $7 \%$ no lo sabían.

No se encontró ninguna otra relación estadística. 


\section{Discusión}

El objetivo de este estudio fue analizar la incidencia, características y horario de las caídas de los pacientes con ERCA en tratamiento con HD fuera de la unidad de diálisis, es decir que no coincidiera con el tiempo de la sesión de HD.

En nuestros resultados hemos podido observar que las caídas del paciente en HD durante el tiempo que no permanecen en la unidad de diálisis presentan una incidencia del $45 \%$. Este dato es similar al comunicado por otros autores, que encuentran una incidencia del $47 \%{ }^{14,16}$.

En cuanto a la influencia de la edad en las caídas, los pacientes que se habían caído son mayores que los que no habían sufrido ninguna caída, si bien estas diferencias no fueron estadísticamente significativas. Respecto al género, hemos encontrado que el $37 \%$ de los hombres que participaron en el estudio había sufrido alguna caída, sin embargo, en el caso de las mujeres, son el $61 \%$ las que tuvieron alguna caída. Estos datos coinciden con otros estudios en el que se destaca la edad y el género femenino como factores de riesgo ante las caídas ${ }^{16,17}$ si bien hay que indicar que en nuestro estudio las mujeres representaban solo el $30 \%$ de la muestra.

Es interesante resaltar el papel que juega el tiempo en diálisis, al menos en nuestros pacientes. En efecto, los pacientes que habían tenido alguna caída llevaban más tiempo en HD que los pacientes que nunca han sufrido caídas, siendo estas diferencias estadísticamente significativas. Sin embargo no hemos encontrado bibliografía con la que contrastar estos resultados.

Cuando se analiza, tanto la actividad física que dice realizar el paciente como su capacidad para la AVD, los pacientes que han tenido caídas tienen mayor grado de dependencia y además realizan menor actividad física. Los pacientes independientes solo habían sufrido caídas el $27 \%$; mientras que los pacientes que presentaban dependencia severa se habían caído el $100 \%$. Es evidente el papel que juega esta variable en la responsabilidad de las caídas, pues habitualmente a la dependencia se asocia edad, comorbilidad, variables que por otra parte, han sido asociadas a las caídas del paciente en $\mathrm{HD}^{16,18,19}$. De igual forma, los pacientes que realizan actividad física mínima se cayeron el $61 \%$ y sin embargo, los que realizaban una actividad vigorosa no tuvieron caídas.
Otro aspecto a destacar fueron las características de las caídas, produciéndose la mayoría de ellas de forma accidental (59\%) y el $22 \%$ debida a hipotensión mareo o hipoglucemia, no siendo significativo el rango horario del día en el que se habían caído o la estación del año. Si resulta interesante resaltar que las caídas se producen en mayor medida en el propio domicilio del paciente $(59 \%)$ y en la calle $(22 \%)$, siendo estos resultados similares a los publicados por otros autores 16,19,20. $^{16}$.

Un aspecto relevante de nuestros resultados es el día en el que se produjo la caída respecto a la sesión de HD. Cabría pensar que esta podría estar relacionada con el tiempo que transcurre después de la sesión de diálisis, en el que el paciente se encuentra agotado y en ocasiones con tendencia a la hipotensión. Sin embargo, las caídas se producen mayoritariamente el día de descanso de diálisis (41\%), repartiéndose por igual (22\%) antes y después de la sesión, tal como describen otros estudios, ${ }^{16,20}$.

Las limitaciones del presente estudio han sido la limitación de la muestra, la falta de estudios en nuestro ámbito asistencial y sobre todo, que al recogerse los datos mediante entrevista en la que el paciente tenía que recordar datos retrospectivos de un año, resultó imposible averiguar el número de caídas totales producidas en ese periodo, porque muchos de ellos no se acordaban, y así establecer el número de caídas por paciente y año, como recoge la mayoría de la bibliografía publicada.

\section{Consideraciones prácticas}

Sería recomendable llevar un registro periódico de las caídas de los pacientes de HD fuera de la unidad de diálisis, para así poder establecer el número de caídas por año. Desde el punto de vista clínico, es importante establecer aquellas medidas de prevención recomendables: prevenir a los pacientes, información, educación sanitaria, en los pacientes frágiles, con el perfil que hemos visto en nuestros resultados.

A la vista de nuestros resultados podemos concluir que las caídas de los pacientes en HD son frecuentes en nuestro medio.

Estas caídas están relacionadas con el género femenino, llevar más tiempo en diálisis, tener mayor grado de dependencia o realizar menor actividad física; y se producen mayoritariamente de forma accidental, en el domicilio del paciente 0 en la calle. 
Además, se producen en mayor medida el día de descanso con respecto a la sesión de diálisis, por lo que habrá que enfocar las medidas preventivas al domicilio del paciente en este día, sobre todo.

Recibido: 15 junio 16

Revisado: 20 junio 16

Modificado: 22 junio 16

Aceptado: 27 junio 16

\section{Bibliografía}

1. Otero A, Zunzunegui MV, Rodríguez A, Aguilar MD, Lázaro P. Volumen y tendencias de la dependencia asociada al envejecimiento de la población española. Rev Esp Salud Pública 2004; 78:20113.

2. Romero L, Martín E, Navarro JL, Luengo C. El paciente anciano: demografía, epidemiología y utilización de recursos. En: Sociedad Española de Geriatría y Gerontología. Tratado de geriatría para residentes. Madrid: Marketing \& Comunication; 2006. p. 33-46.

3. Abellán A, Puga MD. Estudio monográfico: La discapacidad en la población española. En: Observatorio de personas mayores, editor. Las personas mayores en España. Informe 2004. Madrid: Ministerio de Trabajo y Asuntos Sociales; 2004. p. 777-809.

4. Walker SR, Wagner M, Tangri N. Chronic kidney disease, frailty, and unsuccessful aging: A review. J Ren Nutr. 2014;24:364-70.

5. de Francisco ÁLM, De la Cruz JJ, Cases A et al. Prevalencia de insuficiencia renal en Centros de Atención Primaria en España: Estudio EROCAP. Nefrología. 2007; 27(3):300-12.

6. Registro de la Sociedad Española de Nefrología. [Consultado el 15/04/16]. Disponible en: http:// www.senefro.org/contents/webstructure/REER Cong_SEN_2015.pdf.

7. De Francisco ALM. Sostenibilidad y equidad del tratamiento sustitutivo de la función renal en España. Nefrología 2011;31(3):241-6.

8. Górriz Teruel JL, Otero González A. Impacto socio sanitario de la enfermedad renal crónica avanzada. Nefrología. 2008; Supl 3: 7-15.

9. Álvarez de Lara MA, Aljama García P. Complicaciones de la hemodiálisis crónica. En: Hernando Avendaño L, Aljama P, Arias M, Caramelo C, Egido J, Lamas S. Nefrología clínica. Madrid: Panamericana, 2009: 922-24.

10. López JM, Jofré R, Verde E. Complicaciones cardiacas en el enfermo en hemodiálisis. En: Jofré $R$, López Gómez JM, Luño J, Pérez García R, Rodríguez Benítez P. Tratado de Hemodiálisis. Segunda edición actualizada. Barcelona. Editorial Médica Jims; 2006: 467-490.

11. da Silva Gama Z, Gómez-Conesa A. Factores de riesgo de caídas en ancianos. Saúde Pública. 2008; 42 (5):946-56.

12. Yamashita T, Douglas N. Risk Factors of Falls in Community-Dwelling Older Adults: Logistic Regression Tree Analysis. The Gerontologist. 2012 Marzo; 0(0), 1-11; doi: 10.1093/geront/gns043.

13. Portilla Franco ME, et al. La fragilidad en el anciano con enfermedad renal crónica. Nefrología. 2016. http://dx.doi.org/10.1016/j.nefro.2016.03.020.

14. Hazin M, Ithamar Silva L. Assessment of the capacity of the Falls Efficacy Scale International in specifying the risk of falling in patients with chronic kidney disease on hemodialysis. Fisioter Pesq. 2013; 00(0):1-6.

15. Índice de Barthel. [Consultado el 30-03-16]. Disponible en: http://www.sas.junta-andalucia.es/library/plantillas/externa.asp?pag=../../publicaciones/datos/139/pdf/CuestionarioEnfermeria2004. pdf.

16. López-Soto PJ, De Giorgi A, Senno E, Ferraresi A. Renal disease and accidental falls: a review of published evidence. BMC Nephrology; 2015; 16:176; doi 10.1186/s12882-015-0173-7. 
17. Kutner NG, Zhang R, Huang, Wasse. H. Falls among hemodialysis patients: potential opportunities for prevention?. Clin Kidney J; 2014; 7 : 257-63; doi: 10.1093/ckj/sfu034.

18. Cook WL, Tomlinson G, Donaldson M et al. Falls and Fall-Related Injuries in Older Dialysis Patients. J Am Soc Nephrol; 2006; 1:1197-204.
19. Desmet C, Beguin C, Swine C, Jadoul M: Falls in hemodialysis patients: Prospective study of incidence, risk factors, and complications. Am J Kidney Dis; 2005; 45:148-53.

20. Roberts R, Jeffrey C, Carlisle G, Brierley E. Prospective investigation of the incidence of falls, dizziness and syncope in haemodialysis patients. Int Urol Nephrol. 2007;39:275-9.

ANEXO 1.

\section{CUESTIONARIO CAÍDAS E INSUFICIENCIA RENAL}

- Sexo: Hombre $\quad$ Mujer

- Edad:

- Tiempo en hemodiálisis:

- Realiza alguna actividad física:

- Ligera Moderada Vigorosa

• ¿Ha sufrido alguna caída en su domicilio este último año?: Si Si N0

- ¿Cómo fue o fueron las caídas?:

- Causa:

- Modo:

- ¿Recuerda la fecha de las caídas (Hora aproximada y mes)?:

- La caída fue:

- El día de la diálisis:

- Antes de la sesión:

- Después de la sesión:

- Durante las 12 horas siguientes a la diálisis:

- El día de descanso:

- ¿En qué lugar de la casa ocurrió la caída?

- ¿Qué medicación toma?

- ¿Relaciona la caída con algo?

- ¿Grado de dependencia (Índice de Barthel:

\section{UNIVERSIDAD

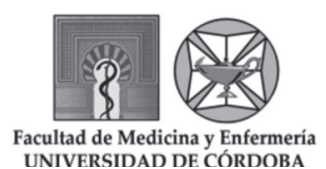

